\title{
Combination of Experts by Classifiers in Similarity Score Spaces
}

\author{
Roberto Tronci ${ }^{1}$, Giorgio Giacinto ${ }^{2}$, and Fabio Roli ${ }^{2}$ \\ 1 AmiLab Laboratorio Intelligenza d'Ambiente, Sardegna Ricerche, 09010 Pula, Italy \\ ${ }^{2}$ DIEE Department of Electrical and Electronic Engineering, University of Cagliari, \\ 09123 Cagliari, Italy \\ \{roberto.tronci, giacinto, roli\}@diee.unica.it
}

\begin{abstract}
The combination of different experts is largely used to improve the performance of a pattern recognition system. In the case of experts whose output is a similarity score, different methods had been developed. In this paper, the combination is performed by building a similarity score space made up of the scores produced by the experts, and training a classifier into it. Different techniques based on the use of classifiers trained on the similarity score space are proposed and compared. In particular, they are used in the framework of Dynamic Score Selection mechanisms, recently proposed by the authors. Reported results on two biometric datasets show the effectiveness of the proposed approach.
\end{abstract}

\section{Introduction}

The combination of ensemble of experts is largely used by the pattern recognition community to improve the performance and the reliability of the classification 1] 2. This combination can be performed at different levels, and with different types of information depending on the pattern recognition problem.

In a number of applications (e.g., biometric authentication, spam filtering, etc.) the task is either to assess if a given test pattern matches to a specific training pattern, or if it belongs or not to a class of patterns (i.e., one-class problems). The task is typically accomplished by "experts" that produce a similarity score. The decision is taken by setting a decision threshold on the score produced by the matching process, so that the outcome is positive if the score is larger than the threshold, while it is negative otherwise. As a consequence, the matching process subdivide patterns into two classes, namely the positive and negative classes. Within the positive and negative classes, the pattern can be further subdivided into "true" and "false" patterns, depending on the fact that the actual class of the pattern is the one assigned by the expert or not. The goal of such experts is to maximize the true positive rate (i.e. the number of positive patterns classified as positive over the total number of positive patterns), while minimizing the false positive rate (i.e. the number of negative patterns classified as positive over the total number of negative patterns). Typically this tradeoff is measured through the use of the Receiver Operating Characteristic curve (ROC), 
which plots the true positive rate against the false positive rate as the decision threshold th varies along the score range [3] 4]. The Area Under the ROC Curve (AUC), and the Equal Error Rate (EER) are two performance measure derived from the ROC that are typically used to compare the performance of different experts. The AUC is the area under the ROC curve, the larger the AUC, the better the ROC. The EER represents the point where the false positive rate and the false negative rate are equal, and a good system should keep this value as small as possible.

It is easy to see that to increase the reliability of the matching process, a number of different matching techniques can be used, thus originating a set of similarity scores. Then, this set of scores can be exploited to produce an ensemble decision, through some functions designed to "fuse" the available scores (e.g., $\max , \min$, linear combination, etc.). The aim of these combination functions is to produce "new" scores whose distributions for the two classes (i.e., positive and negative) exhibit a larger degree of separation than those produced by the individual experts. As a consequence, it is possible to choose decision thresholds allowing to increase the true positive rate at a given false positive rate. In the literature, different types of score combination techniques have been proposed. In particular, some techniques focused on the optimization of the combination parameters in order to maximize some measures of class separability [5] [6] [7].

In this paper, we propose to build a similarity score space, where each component is related to a different expert. This space may allow a better discrimination between true matching pairs and false matching pairs, as different similarity measures can produce false matching on different pairs. A similar representation space has been proposed by the authors in the framework of dynamic classifier selection [8]. Other authors have used this space representation to focus the separation of the classes [9], while similar spaces have been used in specific recognition problems 10. Finally, the formulation of the combination of experts has been also carried our in terms of the joint distribution of the scores produced by the ensemble of experts 7 .

In the similarity score space, we can then train a classifier by using a set of labeled patterns, where the labels are the true outcomes of the matching process (i.e., "positive" or "negative"). When a test pattern is submitted to the system, the similarity score space is built from the ensemble of experts, and the output of the classifier is produced. We propose and compare four uses of the output of the classifier: "crisp" classification, as in a two-class problem; treating the output as a new score, so that the Receiver Operating Characteristic curve can be built; using the "crisp" output to estimate the true outcome in the Dynamic Score Selection (DSS) mechanism (see Section 3) [11; using the continuous output in the generalized DSS mechanism (see Section 3). The aim of this paper is to assess the feasibility of this space for the combination of experts, and to compare the four solution designed to exploit the information provided by classifiers. The paper is organized as follows: Section 2 describes the use of a score space for classification. Section 3 describes the Dynamic Score Selection and its modification. Section 4 reports the experimental phase made 
to compare the different uses of the classifier. Finally the conclusion are outlined in Section 5 .

\section{The Similarity Score Space of an Ensemble of Two-Class Experts}

As stated in the introduction, it has been proved that the combination of different experts can improve the performance of a pattern recognition system [1] [2].

Let $E=\left\{E_{1}, E_{2}, \ldots E_{j} \ldots E_{N}\right\}$ be a set of $N$ experts and $X=\left\{x_{i}\right\}$ be a set of patterns to be tested. Let also $s_{i j}$ be the score produced by the expert $E_{j}$ for the pattern $x_{i}$. Let $S_{j}$ be the set of all the scores produced by an expert $E_{j}$ for all the patterns $X$. Generally a combination function of experts takes as input the similarity scores estimated for a pattern and combines them in some way to obtain a combined score as shown in Figure 1

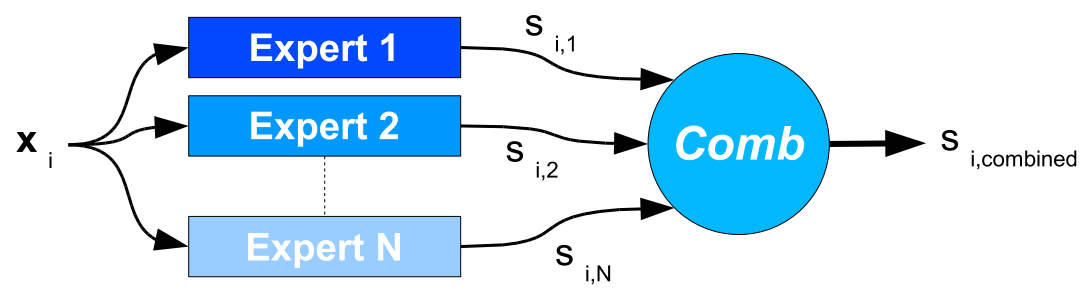

Fig. 1. General schema of an expert combination system

These combination functions produce a "new" score usually through a mathematical function of the set of scores estimated by the $\mathrm{N}$ experts, e.g., max, min, linear combination, etc.

In this paper we use these scores not as an input of a combination function, but as a "similarity" feature vector to represent each input pattern, whose components are the scores assigned to that pattern by the ensemble of experts $E_{j}$. Thus, for each pattern $x_{i}$ the following feature vector $v_{i}=\left\{s_{i 1}, \ldots s_{i j} \ldots s_{i N}\right\}$ can be built [8] 9] 10].

Using these vectors, we are able to represent the patterns in a similarity score space. An example of the use of this space is shown in Figure 2 In this figure a $2 \mathrm{D}$ and a $3 \mathrm{D}$ spaces are shown, where the $2 \mathrm{D}$ space is related to an ensemble of two experts, while the $3 \mathrm{D}$ space is obtained from the $2 \mathrm{D}$ space by adding another expert. In the figure, the distribution of positive (colored in blue) and negative (colored in red) patterns are reported. Thus it follows that we transformed a matching problem (or a one-class problem) into a two-class problem.

From the examples in the two figures it is easy to see that the increase of the number of experts produces a larger separation of patterns belonging to different classes. It is also evident that generally the separation surface of the two classes is not linear. To estimate this separation surface, a classifier can be trained, and its output can be used in two ways: 

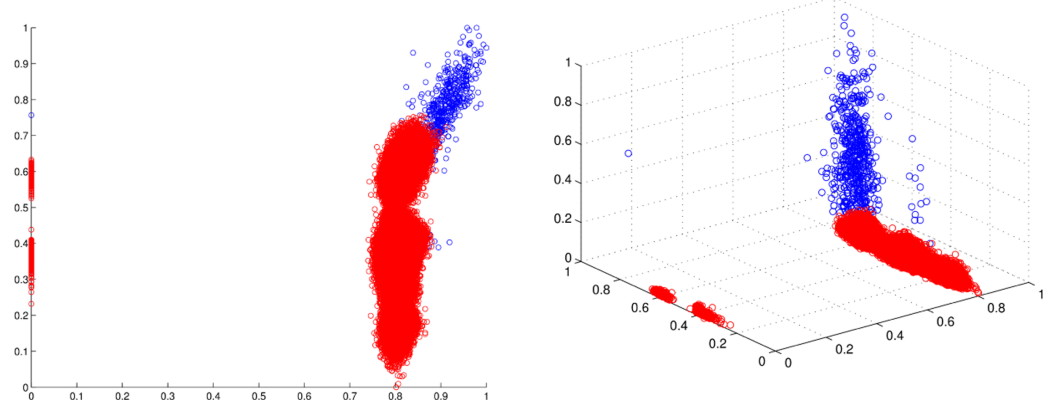

Fig. 2. Example of the distribution of scores in a space in a two-dimensional and threedimensional space. The three-dimensional space is obtained by adding a dimension to the two-dimensional space. By increasing the number of dimensions, the separability increases. The positive class is represented in blue, while the negative class, in red.

1. Using directly the class assigned by the classifier ("crisp" classification)

2. Using the continuous output of the classifier as a support for the combination of the scores of the ensemble of experts

In the first case, the output of the classifier is simply the estimated class. The performance of the system can then be measured in terms of the accuracy of classification, i.e. the "true positive" rate, and the "false positive" rate.

In the second case, we can consider the output of a classifier as the results of the combination produced by the ensemble of experts, thus the performance can be expressed using the same measures used for the individual experts $E_{j}$, i.e. the Receiver Operating Characteristic curve (ROC), the Area Under the ROC Curve (AUC), and the Equal Error Rate (EER).

It is easy to see that if the "crisp" classification always exhibits an accuracy of $100 \%$, then the direct use of the class assigned by a classifier is more advantageous than the use of the continuous output of the classifier. In practical application it is very hard to reach a $100 \%$ accuracy, thus it is worth investigating further exploitation of the output of the classifier. To this end, we propose two algorithms based on "crisp" outputs, and continuous outputs, respectively. These algorithms are based on the Dynamic Score Selection algorithm [11, whose basic concepts are recalled in the following Section.

\section{Dynamic Score Selection Based on Classifiers in Similarity Spaces}

Dynamic Score Selection (DSS) techniques are based on the concept of the ideal score selector [11] who aims to select one score from those assigned to the pattern by the ensemble of experts. In particular, ideal selection has been defined as the process of selecting for each pattern the maximum score among those assigned 
to the pattern by the ensemble if it is a positive pattern, and the minimum score if it is a negative pattern.

$$
s_{i, *}=\left\{\begin{array}{l}
\max \left\{s_{i j}\right\} \text { if } x_{i} \text { is a positive pattern } \\
\min \left\{s_{i j}\right\} \text { if } x_{i} \text { is a negative pattern }
\end{array}\right.
$$

It can be proved that the above ideal score selector exhibits a better ROC curve than every single ROC curve of the individual experts used in the combination, and consequently a larger AUC [11.

We propose to implement the DSS by exploiting either the "crisp" output of a classifier or the continuous output, when available. When a "crisp" output is available, it can be used as an estimation of the state of nature of a test pattern (i.e., if it is positive or negative), and the score is chosen according to Equation (11).

The proposed DSS algorithm is made up of the following steps:

1. Construct the $N$-dimensional vector space made up of the score outputs of the $N$ available experts

2. Train a classifier $C$ in the $N$-dimensional vector space using a set of labeled patterns

3. Classify test patterns with the classifier $C$

4. Select the score $s_{\text {sel }}$ based on the output class of classifier $C$ as follows

$$
s_{\text {sel }}= \begin{cases}\max _{j}\left(s_{i j}\right) & \text { if class }=\text { positive } \\ \min _{j}\left(s_{i j}\right) & \text { if class }=\text { negative }\end{cases}
$$

We call such a DSS algorithm as "crisp" DSS as it exploit only the "crisp" decisions made by the classifier.

If we consider the continuous outputs provided by the classifier, it is possible to develop a Dynamic Score Combination algorithm, that can be considered as a generalization of the basic DSS algorithm:

$$
s_{i, d s s}=\beta_{i} \cdot \max _{j}\left(s_{i j}\right)+\left(1-\beta_{i}\right) \cdot \min _{j}\left(s_{i j}\right)
$$

where $\beta_{i} \in[0,1]$ represents the continuous outputs of a classifier. If $\beta=1$, than the pattern is estimated as positive, otherwise if $\beta=0$ the pattern is estimated as negative. It is easy to see that the formulation in Equation 2 embodies the "crisp" DSS.

Thus, the output of the classifier can be used either to perform a "hard" selection of one of the available scores (Equation (1)), or a "soft" selection, based on the linear combination of the max and min scores by a dynamic weight that is the output of the classifier (Equation (2) ).

\section{Experiments}

The aim of the reported experiments is to show the capabilities of similarity score space for the combination of experts, and to compare the four different 
uses of classifiers trained on a similarity score space, i.e., the "crisp" output, the continuous output, the "crisp" DSS, and the generalized DSS. Experiments have been performed using two multimodal biometric authentication datasets.

Firstly, we will compare the performance of "crisp" classifiers with those of the DSS based on "crisp" outputs and the generalized DSS, in terms of false positive and false negative rates. The dataset used for these experiments is made up by the scores obtained during the third Fingerprint Verification Competition 1 (FVC2004) 12. From the FVC2004, we use both the Open category (made up of 41 high performance experts), and the Light category (made up of 26 low performance experts). The fingerprint images were acquired using four sensors. For each sensor and for each expert, 2800 of positive and 4950 of negative scores are available. For the details on how the scores where obtained and normalized, the reader is referred to [12. This database is not freely available, so our algorithms were executed at the Biometric Systems Lab (University of Bologna) which organizes the competition.

Then, the performances of the "crisp" DSS, the generalized DSS, and that of the continuous output of classifiers will be compared in terms of AUC and EER. In these experiments the dataset used is the Biometric Scores Set Release 1 of the National Institute of Standards and Technology 2 (NIST). The dataset contains similarity scores from two face recognition systems and one fingerprint system on left and right index fingerprints. For aim of this paper, the set containing the face and the fingerprint systems were used. In this multimodal dataset are present 517 subjects. The scores were normalized using the Min-Max rule.

In order to create a training set for the classifiers, we randomly divided the set of scores into four subsets of the same size for both the NIST and the FVC2004 datasets. Each of the four subsets has been used for training, while the remaining three subsets have been used for testing. Using this partitioning of the dataset, we performed an exhaustive multi-modal combination experiment for the NIST dataset, and an exhaustive multi-algorithm combination experiment for the FVC2004 dataset for each sensor.

In Figures (3, 4), the graphs related to experiments carried out on the FVC2004 dataset are shown. For these experiments four classifiers have been used: the kNearest Neighbor (kNN), the Linear Discriminant Classifier (LDC), the Quadratic Discriminant Classifier (QDC), and a Parzen classifier. The comparison is made in the following way, as the "crisp" outputs of classifiers are obtained by fixing a decision threshold at 0.5 (the output of classifiers is normalized in the range $[0,1]$ ):

- each classifier is trained on the four subdivision of the dataset described above.

- for each trained classifier $C$ the $F M R$ and $F N M R$ are computed on the test set as follows considering the estimated state of nature

$F M R=$ negatives classified as positives / total negatives

FNMR = positives classified as negatives / total positives

\footnotetext{
${ }^{1}$ Web site: http://bias.csr.unibo.it/fvc2004/

${ }^{2}$ Web site: http://www.itl.nist.gov/iad/894.03/biometricscores/
} 


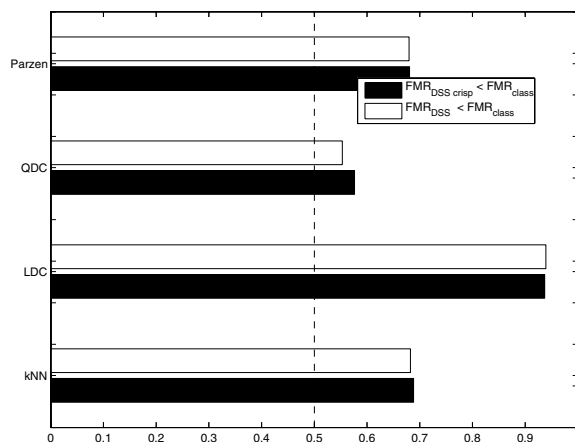

(a)

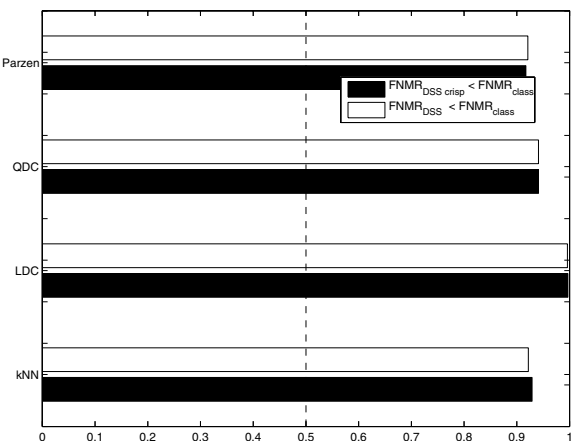

(b)

Fig. 3. Comparison between the "crisp" classifier and the "crisp" and generalized DSS using the Open FVC2004 dataset. In white are reported the occurrences when the generalized DSS exhibits lower errors than the classifier. In black are reported the occurrences when the "crisp" DSS exhibits lower errors than the classifier. In (a) the threshold for the DSS is chosen to obtain $F N M R_{\text {class }}=F N M R_{D S S}$. In (b) the threshold is chosen to have $F M R_{\text {class }}=F M R_{D S S}$.

- for each classifier a "crisp" and generalized DSS is built using the output of the classifier

- for each classifier and subdivision, the value of $F M R$ is compared to that produced by the related DSS when the value of $F N M R$ of the DSS is equal to the value of $F N M R$ produced by the classifier.

- for each classifier and subdivision, the value of $F N M R$ is compared to that produced by the related DSS when the value of FMR of the DSS is equal to the value of $F M R$ produced by the classifier.

In Figure (3) the results on the Open dataset are shown, while in Figure (4) the results on the Light dataset are reported. The bars represent the percentage of cases in which the DSS outperformed the "crisp" classifier. From these figures it is clear that the use of the classifier as a component of a Dynamic Score Selector is generally preferable to the direct use of the "crisp" classifier. Moreover, the analysis of the figures clearly shows that this advantages depends on the classifier used, when the comparison is made by fixing the value of FNMR. The more simple the classifier, the better the performance of the DSS with respect to that of the classifier, e.g. the performance of LDC are very low with respect to the DSS based on LDC, while in the case of QDC the performance are quite similar. On the other hand, if the value of FMR is fixed, the DSS always outperforms the "crisp" classifier in terms of FNMR. Thus, the DSS algorithm allows attaining a better tradeoff of FMR and FNMR than that attained by a "crisp" classifier. The comparison between the two versions of the DSS shows that the "crisp" version slightly performs better than the generalized version when the FNMR value is fixed. When the value of FMR is fixed, the two versions of DSS show a quite similar performance. However, a better comparison of the performance of 


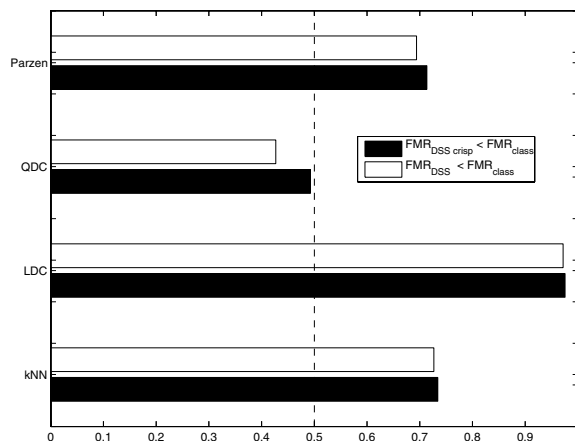

(a)

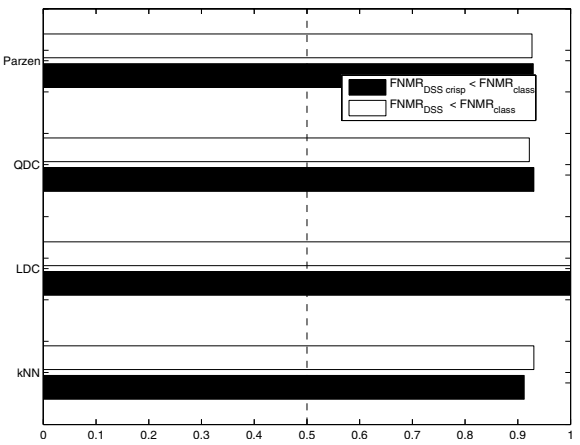

(b)

Fig. 4. Comparison between the "crisp" classifier and the "crisp" and generalized DSS using the Light FVC2004 dataset. In white are reported the occurrences when the generalized DSS exhibits lower errors than the classifier. In black are reported the occurrences when the "crisp" DSS exhibits lower errors than the classifier. In (a) the threshold for the DSS is chosen to obtain $F N M R_{\text {class }}=F N M R_{D S S}$. In (b) the threshold is chosen to obtain $F M R_{\text {class }}=F M R_{D S S}$.

Table 1. Mean and standard deviation of AUC as the dimension of the similarity score space increases. All the possible combination of 2,3 and 4 experts are used.

\begin{tabular}{cccc} 
AUC & 2 Experts & 3 Experts & 4 Experts \\
\hline \hline$L D A$ & $0.9887( \pm 0.0073)$ & $0.9945( \pm 0.0040)$ & $0.9977( \pm 0.0019)$ \\
$D S S L D C$ “crisp" & $0.9743( \pm 0.0103)$ & $0.9728( \pm 0.0088)$ & $0.9744( \pm 0.0055)$ \\
$D S S k$ - $N$ “crisp" & $0.9859( \pm 0.0068)$ & $0.9917( \pm 0.0050)$ & $0.9955( \pm 0.0023)$ \\
$D S S Q D C$ “crisp" & $0.9833( \pm 0.0112)$ & $0.9919( \pm 0.0086)$ & $0.9966( \pm 0.0050)$ \\
$D S S S V M$ “crisp" & $0.9789( \pm 0.0094)$ & $0.9799( \pm 0.0077)$ & $0.9799( \pm 0.0000)$ \\
$D S S L D C$ & $0.9752( \pm 0.0103)$ & $0.9741( \pm 0.0087)$ & $0.9760( \pm 0.0051)$ \\
$D S S k-N N$ & $0.9936( \pm 0.0065)$ & $0.9987( \pm 0.0016)$ & $\mathbf{0 . 9 9 9 8}( \pm \mathbf{0 . 0 0 0 1})$ \\
$D S S Q D C$ & $0.9901( \pm 0.0083)$ & $0.9964( \pm 0.0039)$ & $0.9991( \pm 0.0008)$ \\
$D S S$ SVM & $0.9946( \pm 0.0064)$ & $\mathbf{0 . 9 9 9 6}( \pm \mathbf{0 . 0 0 0 4})$ & $\mathbf{0 . 9 9 9 8}( \pm \mathbf{0 . 0 0 0 2})$ \\
$L D C$ est & $0.9891( \pm 0.0076)$ & $0.9947( \pm 0.0038)$ & $0.9973( \pm 0.0017)$ \\
$k-N N$ est & $0.9699( \pm 0.0226)$ & $0.9928( \pm 0.0075)$ & $0.9973( \pm 0.0051)$ \\
$Q D C$ est & $\mathbf{0 . 9 9 5 3}( \pm \mathbf{0 . 0 0 4 7})$ & $0.9985( \pm 0.0005)$ & $0.9986( \pm 0.0001)$ \\
$S V M$ est & $0.9049( \pm 0.0600)$ & $0.9360( \pm 0.0191)$ & $0.9996( \pm 0.0003)$ \\
\hline
\end{tabular}

different versions of the DSS is made in the following Tables in terms of AUC and EER.

Tables (112) show the comparison of performances of the "crisp" DSS, the generalized DSS, the output of the classifiers treated as a "new" score, and a "trained fusion" rule based on a linear score combination whose weights are computed by Linear Discriminant Analysis (LDA) 9 [13. These results have been obtained on the NIST dataset, and the performance are reported in terms of AUC and EER, for different number of experts combined (i.e., 2, 3, and 4 
Table 2. Mean and standard deviation of EER as the dimension of the similarity score space increases. All the possible combination of 2,3 and 4 experts are used.

\begin{tabular}{cccc} 
EER & 2 Experts & 3 Experts & 4 Experts \\
\hline \hline LDA & $0.0431( \pm 0.0166)$ & $0.0296( \pm 0.0123)$ & $0.0200( \pm 0.0068)$ \\
$D S S$ LDC “crisp" & $0.0634( \pm 0.0162)$ & $0.0647( \pm 0.0148)$ & $0.0632( \pm 0.0098)$ \\
$D S S$ k-NN “crisp" & $0.0377( \pm 0.0153)$ & $0.0236( \pm 0.0072)$ & $0.0199( \pm 0.0067)$ \\
$D S S Q D C$ “crisp" & $0.0453( \pm 0.0228)$ & $0.0243( \pm 0.0182)$ & $0.0138( \pm 0.0124)$ \\
$D S S S V M$ “crisp" & $0.0525( \pm 0.0170)$ & $0.0497( \pm 0.0140)$ & $0.0547( \pm 0.0000)$ \\
$D S S L D C$ & $0.0629( \pm 0.0164)$ & $0.0642( \pm 0.0149)$ & $0.0632( \pm 0.0098)$ \\
$D S S$ k-NN & $0.0261( \pm 0.0175)$ & $0.0104( \pm 0.0053)$ & $0.0071( \pm 0.0025)$ \\
$D S S$ QDC & $0.0324( \pm 0.0198)$ & $0.0147( \pm 0.0092)$ & $0.0097( \pm 0.0044)$ \\
$D S S$ S VM & $\mathbf{0 . 0 2 1 2}( \pm \mathbf{0 . 0 1 8 2})$ & $\mathbf{0 . 0 0 4 8}( \pm \mathbf{0 . 0 0 2 6})$ & $\mathbf{0 . 0 0 4 0}( \pm \mathbf{0 . 0 0 2 4})$ \\
$L D C$ est & $0.0429( \pm 0.0164)$ & $0.0300( \pm 0.0116)$ & $0.0206( \pm 0.0069)$ \\
$k-N N$ est & $0.0340( \pm 0.0238)$ & $0.0101( \pm 0.0051)$ & $0.0058( \pm 0.0033)$ \\
$Q D C$ est & $0.0214( \pm 0.0161)$ & $0.0094( \pm 0.0050)$ & $0.0070( \pm 0.0013)$ \\
$S V M$ est & $0.0965( \pm 0.0582)$ & $0.0640( \pm 0.0191)$ & $\mathbf{0 . 0 0 4 0}( \pm \mathbf{0 . 0 0 2 4})$ \\
\hline
\end{tabular}

experts). Four classifiers have been used, namely, the k-Nearest Neighbor $(\mathrm{kNN})$, the Linear Discriminant Classifier (LDC), the Quadratic Discriminant Classifier (QDC), and the Support Vector Machine with a radial basis kernel. All the classifier are trained using the dataset subdivision described above, and all the possible combination of experts had been performed.

The results reported in Tables (112) confirm one of the initial intuition of the paper: the larger the dimension of the score space, the better the separability. In fact all the methodologies, but the LDC, increase their performance as the number of scores for pattern increases. This aspect is more evident when we take into account the results obtained with the estimation of the SVM, as the increasing number of scores per pattern leads to a high increase in performance. If we compare the performance of different version of DSS and those of the output of the classifier, it is easy to see that the generalized version of the DSS outperform the "crisp" DSS both in terms of AUC and EER. Moreover, the generalized version of the DSS outperforms the LDA in terms of AUC and EER when the $\mathrm{kNN}$, the QDC, and the SVM are considered. The performances attained by the output of the classifier used as a "new" score are quite similar to those of the generalized DSS. However, the best results have been produced by the generalized DSS when the SVM classifier is used.

\section{Conclusions}

In this paper we showed the use of a similarity score space to combine different experts. In this space a classifier can be trained and its output used to improve the performance. In particular, we showed different ways of using the trained classifier: using directly its "crisp" classification, using the continuous output of the classifier either as a new score, or as a support for Dynamic Score Selection mechanisms. Reported experiments showed that the best use of the output 
of classifiers built in similarity score spaces is with Dynamic Score Selection mechanisms, as they allow obtaining lower errors than those obtained through the "crisp" classification. In particular, the best performances are provided by a generalized Dynamic Score Selection mechanism based on SVM. Moreover, reported results also show that the larger the dimension of the similarity score space (i.e., the larger the number of experts in the ensemble), the better the performance.

\section{References}

1. Kittler, J., Hatef, M., Duin, R., Matas, J.: On combining classifiers. IEEE Transactions on Pattern Analysis and Machine Intelligence 20(3), 226-239 (1998)

2. Kuncheva, L.: Combining Pattern Classifiers: Methods and Algorithms. John Wiley \& Sons, Chichester (2004)

3. Bradley, A.: The use of the area under the ROC curve in the evaluation of machine learning algorithms. Pattern Recognition 30(7), 1145-1159 (1997)

4. Fawcett, T.: An introduction to roc analysis. Pattern Recognition Letters 27(8), $861-874(2006)$

5. Marrocco, C., Molinara, M., Tortorella, F.: Exploiting AUC for optimal linear combinations of dichotomizers. Pattern Recognition Letters 27(8), 900-907 (2006)

6. Ross, A., Nandakumar, K., Jain, A.: Handbook of Multibiometrics. Springer, Heidelberg (2006)

7. Nandakumar, K., Chen, Y., Dass, S., Jain, A.: Likelihood ratio-based biometric score fusion. IEEE Trans. on PAMI 30(2), 342-347 (2008)

8. Giacinto, G., Roli, F.: Dynamic classifier selection based on multiple classifier behaviour. Pattern Recognition 34(9), 179-181 (2001)

9. Ross, A., Jain, A.K.: Information fusion in biometrics. Pattern Recognition Letters 24, 2115-2125 (2003)

10. Lumini, A., Nanni, L.: Advanced methods for two-class pattern recognition problem formulation for minutiae-based fingerprint verification. Pattern Recognition Letters 29(2), 142-148 (2008)

11. Tronci, R., Giacinto, G., Roli, F.: Dynamic Score Selection for Fusion of Multiple Biometric Matchers. In: ICIAP 2007, pp. 15-20 (2007)

12. Maio, D., Maltoni, D., Cappelli, R., Wayman, J., Jain, A.: FVC2004: Third Fingerprint Verification Competition. In: Zhang, D., Jain, A.K. (eds.) ICBA 2004. LNCS, vol. 3072, pp. 1-7. Springer, Heidelberg (2004)

13. Duda, R., Hart, P., Stork, D.: Pattern Classification. John Wiley \& Sons, Chichester (2001) 